\title{
La relation enseignant/apprenti traducteur dans le processus de formation à la traduction. Étude de cas: les mémoires de fin deétudes
}

\author{
Ileana Neli EIBEN \\ Université de l'Ouest, Timişoara \\ Roumanie
}

Résumé: Dans cet article, nous nous proposons de présenter les difficultés que les étudiants en langues étrangères appliquées, plus particulièrement ceux de l'Université de l'Ouest de Timişoara, sont tenus à résoudre pendant la rédaction de leurs mémoires de fin d'études. Nous nous interrogeons sur la façon dont l'enseignant peut répondre aux attentes des apprentis traducteurs lors d'un moment-clé de leur vie: fin de leur formation universitaire et début de l'activité professionnelle. L'enseignant joue un róle important: il les guide de près dans leurs activités tout en leur enseignant à devenir autonomes. Il les stimule à prendre confiance dans leur capacité d'apprentissage qui leur servira dans l'activité professionnelle et dans la vie. En collaborant avec les étudiants, il les aide à faire face aux défis de la nouvelle société caractérisée par l'instabilité de l'emploi.

Mots-clés: mémoire de fin d'études, commentaire de traduction, autorévision, lecture traductologique, collaboration enseignant/apprenti traducteur.

\begin{abstract}
This article intends to present the difficulties that the Applied Foreign Languages students, especially those at the West University of Timişoara, experience when writing their Bachelor's theses. In addition, it reflects on how teachers manage to meet apprentice translators' expectations in a key moment in their lives, i.e. when graduating from university and beginning their professional activity. Teachers play a significant role: they guide students closely while helping them to become independent. They collaborate with students and stimulate them into gaining a certain confidence and independence that will help them in their professional activities and in their lives. In collaborating with students, teachers help them face the challenges of a new society, characterised by employment instability.
\end{abstract}

Key-words: Bachelor's Thesis, translation commentary, self-revision, translation reading, teacher-student collaboration.

\section{Introduction}

L'objectif principal de notre article est de présenter le travail de traduction et de rédaction que les étudiants en langues étrangères appliquées entreprennent à la fin du premier cycle de leur formation 
universitaire. Pour ce faire, nous essayerons de mettre à profit notre expérience acquise en tant qu'enseignant au département LEA de l'Université de l'Ouest de Timişoara et en tant que cotuteur de mémoire de licence.

Un autre objectif de notre contribution serait de mettre en évidence la façon dont l'enseignant répond aux attentes des apprentis traducteurs lors d'un moment-clé de leur vie: fin (pour certains) de la formation universitaire et début de l'activité professionnelle. Comment peut-il les aider à faire face aux défis de la nouvelle société caractérisée par l'instabilité de l'emploi, la mondialisation et l'essor de nouvelles technologies qui évoluent à des rythmes galopants?

Il y a deux raisons qui nous ont déterminée à aborder ici la problématique des mémoires de licence. Tout d'abord, ce travail représente l'aboutissement de trois années d'étude. Les étudiants ont ainsi l'occasion d'une part, de valoriser les connaissances déjà acquises et, d'autre part, d'enrichir leur expérience pour rédiger des travaux de recherche similaires pour le cas où ils poursuivront leurs études par un master de spécialité. Ensuite, le mémoire est la preuve de la maturité du candidat, de sa capacité de rédiger un texte scientifique, de le présenter devant un jury et de répondre aux questions qui lui sont posées.

Avant de focaliser notre attention sur les mémoires de fin d'études codirigés, il n'est pas superflu de rappeler succinctement les caractéristiques générales de ces productions scientifiques.

Réaliser un mémoire signifie:

-choisir un sujet précis,

-se documenter sur un thème choisi,

-être capable d'ordonner des documents,

-analyser le sujet choisi à la lumière des documents consultés,

-donner une forme cohérente à toutes les réflexions précédentes,

-faire en sorte que le texte soit lisible et, si nécessaire, qu'il serve de point de départ à une recherche future (Eco 2006, 23).

En d'autres mots, réaliser un mémoire signifie: apprendre à mettre de l'ordre dans ses propres idées, ordonner des documents, rédiger un texte cohérent, être original tout en respectant des règles, et stimuler par sa contribution d'autres recherches futures sur le même sujet d'étude.

\section{Présentation des mémoires codirigés}

Les apprentis traducteurs dont nous nous sommes occupée ont choisi de faire un commentaire de traduction en travaillant sur des textes économiques ou sur différentes parties de l'ouvrage de Jean Delisle, La traduction raisonnée (2003). Ces derniers s'inscrivent dans un projet plus ample et ils continuent chaque fois le travail de leurs prédécesseurs. 
À l'Université de l'Ouest de Timişoara, les mémoires de licence se composent en général de trois parties: la première partie théorique est réservée aux particularités du texte à traduire, la deuxième partie comprend la traduction proprement dite et la troisième partie prend la forme d'un commentaire de traduction à partir des difficultés de traduction que l'apprenant a dû affronter et dépasser lors du processus de traduction.

\section{3. Élaboration des mémoires de fin d'études}

La recherche scientifique des étudiants dont nous avons coordonné les mémoires comprend deux moments clés. Dans un premier temps ils doivent traduire un texte de spécialité (ils ont le choix entre plusieurs domaines: économie, droit, médecine, traductologie, etc.). Les livres dont ils extraient les fragments à traduire doivent être de date assez récente, c'est-à-dire publiés pendant les dix dernières années. Dans un deuxième temps, après avoir mené une activité de recherche terminologique et traductologique, ils entament la rédaction de leur mémoire.

\subsection{Traduction d'un texte de spécialité}

La mise en roumain du texte-source en français constitue la première étape de leur travail. Elle comprend trois moments-clés: la traduction proprement dite, l'autorévision (Plassard 2007, 225) et la révision du texte par l'enseignant.

Lorsqu'ils procèdent à la traduction, le premier obstacle que les apprentis traducteurs doivent dépasser est la longueur du texte: jamais auparavant ils n'ont eu à traduire tout seuls une cinquantaine de pages. Il y en a qui commencent à la fin de la deuxième année d'étude pour avoir le temps de parcourir le texte d'une manière approfondie. Il y en a aussi qui s'y mettent au dernier moment au risque de bâcler ou de rater leur mémoire.

Un autre aspect susceptible d'entraver leur activité de traduction serait une compétence linguistique insuffisante pour bien comprendre le texte-source en français. Pour suppléer à ces carences, ils font appel aux dictionnaires qu'ils prennent pour des auctoritates et non pas pour des outils. Assez souvent ils n'arrivent pas à faire le tri pour en extraire l'information la plus adéquate et ils finissent par obtenir du faux sens, du contre sens ou même du non sens.

Lors de l'étape de traduction nous intervenons peu et seulement si les étudiants sollicitent notre aide: par exemple, s'ils ont des difficultés à comprendre certains fragments du texte-source, à trouver des structures équivalentes en langue-cible ou à choisir une stratégie de traduction adéquate. Nous les aidons alors à se rappeler des notions acquises auparavant et à les mettre en œuvre. De même, en fonction du texte à traduire, nous leur fournissons des pistes pour mener des recherches individuelles. Cette implication limitée de l'enseignant 
permet à l'étudiant d'acquérir une certaine autonomie dans son travail. D'ailleurs, elle sera suivie par l'autorévision, étape que nous considérons absolument nécessaire. Nous recommandons aux apprentis traducteurs de relire le texte eux-mêmes. Cette activité de lecture sera suivie dans un deuxième temps par une intervention sur le texte.

Il est important que l'apprenti traducteur jette un regard critique sur son travail et qu'il s'habitue à ne pas remettre un texte mal rédigé. La lecture de son propre texte lui offre l'occasion d'acquérir une compétence critique qui « conjugue l'aptitude à se relire, autrement dit à savoir dissocier les langues d'une part et les représentations mentales d'autre part, à savoir diagnostiquer les sources de dysfonctionnement textuel, autrement dit être à même de porter un jugement métalinguistique sur sa propre production et enfin à savoir y remédier, c'est-à-dire disposer d'une palette d'outils d'intervention sur le texte " (Plassard 2007, 225).

En ce qui concerne le type de lecture, nous recommandons une « lecture pointage » (Plassard 2007, 227) qui porte sur l'exhaustivité du texte. L'apprenti traducteur doit alors faire attention:

- au respect de l'intégralité du texte ;

- à la « vérification de tous les éléments textuels et paratextuels [...], de la graphie des noms propres à la siglaison en passant par les critères purement typographiques » (Plassard 2007, 227).

Lors de l'étape de lecture l'apprenti traducteur change de statut: il devient le premier lecteur de son texte, « il s'inscrit lui-même dans la communauté des lecteurs ou du pôle de lecture auquel le texte [...] fraîchement rédigé est destiné, lecteurs dont il apparait en quelque sorte comme le prototype » (Plassard 2007, 222).

En endossant le statut de lecteur, l'apprenti traducteur peut anticiper la lecture de sa propre production faite par un tiers. Il en est aussi le premier récepteur se retrouvant dans la situation de vérifier s’il est possible de comprendre le contenu à partir de seule la traduction, le produit de son travail. La compréhension de sa propre production en langue-cible facilite la future intervention sur le texte qui présuppose " de reconnaître les 'remèdes' aux dysfonctionnements constatés, remèdes qui consistent notamment en la faculté de reformulation, de paraphrase, de jeu avec un éventail de structures syntaxiques à la disposition du scripteur » (Plassard 2007, 226).

Il est certain que l'apprenti traducteur n'est pas en mesure de saisir et de corriger toutes les erreurs qu'il a faites dans sa traduction. Cependant, nous considérons qu'il est capable d'en améliorer et la forme et le contenu. La distance temporelle qui sépare les deux moments lui permet de se détacher du texte et de la langue-source dont il était imprégné lorsqu’il traduisait. Grâce à ce détachement il pensera à respecter l'intégralité du texte et il mobilisera des ressources linguistiques lui permettant une gamme plus large de formulations en langue-cible, en l'occurrence en langue maternelle. 
Nous accordons beaucoup d'attention à l'autorévision. En effet, la lecture critique appliquée par l'auteur à son propre texte est «l'un des meilleurs moyens dont dispose tout chercheur pour améliorer celuici » (Gile 1995, 6).

Le travail de l'apprenti traducteur terminé, nous commençons à réviser et à corriger le texte. Nous réalisons une confrontation de la traduction à l'original en vérifiant si l'étudiant a restitué intégralement le texte-source et s'il en a respecté les aspects formels: structure des paragraphes, mise en page, titres, sous-titres, etc. Bien que, lors de l'autorévision, nous recommandions une lecture exhaustive (v. supra), il nous arrive souvent de constater qu'il reste encore du travail à faire.

\section{Exemple 1:}

\begin{tabular}{|l|l|}
\hline \multicolumn{1}{|c|}{ Texte-source } & \multicolumn{1}{|c|}{ Texte-cible } \\
\hline Source: Société canadienne des postes & Sursa: Societatea canadiană de poştă \\
Genre de publication: Brochure & Fragment absent \\
illustrée contenant 25 timbres-poste & \\
Date de parution: 1990 & Data apariției: 1990 \\
Domaine: Histoire des postes (Delisle & Domeniu: Istoria poştei \\
2003, p. 132-133) & \\
\hline
\end{tabular}

\section{Exemple 2:}

\begin{tabular}{|c|c|}
\hline Texte-source & Texte-cible \\
\hline $\begin{array}{l}\text { Les termes suivants se prêtent } \\
\text { bien à des exercices d'interprétation } \\
\text { lexicale qui obligent le traducteur à } \\
\text { chercher le mot juste et à varier son } \\
\text { expression: aggressive, approach, } \\
\text { available, challenge, clinic, control, } \\
\text { corporate, developement, dollar, } \\
\text { facilities, identify, involve, issue, } \\
\text { jurisdiction, -minded, -conscois si - } \\
\text { oriented, pattern, policy, problem, } \\
\text { procedure, system, type (Delisle } \\
\text { 2003.235). }\end{array}$ & $\begin{array}{l}\text { Următorii termeni se pretează } \\
\text { bine în exerciții de interpretare } \\
\text { lexicală ce îl obligă pe traducător să } \\
\text { caute cuvântul potrivit şi să işi } \\
\text { schimbe exprimarea: aggressive, } \\
\text { approach, available, challenge, clinic, } \\
\text { control, corporate, developement, } \\
\text { dollar, facilities, identify, involve, } \\
\text { issue, jurisdiction, -minded, -conscois } \\
\text { si - oriented, pattern, policy, problem, } \\
\text { procedure, system, type. }\end{array}$ \\
\hline
\end{tabular}

Dans le premier exemple ci-dessus, on peut remarquer qu'une partie du texte-source n'a pas été traduite en roumain. En italique, nous avons indiqué l'emplacement de l'unité manquante en suggérant à l'apprenti traducteur de revenir à l'original et de revoir sa traduction. Dans le deuxième exemple, nous avons constaté que les mots anglais reportés dans le texte-cible n'ont pas été mis en évidence. Alors, nous avons inséré des commentaires en recommandant que la graphie du texte-source soit respectée.

De même, une compétence linguistique limitée en langue étrangère peut entraver l'accès au sens. Parfois, en lisant la traduction 
nous révélons des erreurs qui indiquent le plus souvent une compréhension insuffisante d'une partie du texte-source. Alors, nous revenons à l'original pour corriger ces fautes et comprendre les facteurs qui ont déterminé une formulation défectueuse en langue-cible.

Exemple 3:

\begin{tabular}{|l|l|}
\hline \multicolumn{1}{|c|}{ Texte-source } & \multicolumn{1}{|c|}{ Texte-cible } \\
\hline Dès les années 1730, des maisons de & Începându cu anul 1730, ease de \\
relais assurent la liaison entre les & sehimb hanuri de poștă asigură \\
illots de civilisation disséminés sur le & $\begin{array}{l}\text { legătura între insulițele de civilizație } \\
\text { territoire de la Nouvelle-France } \\
\text { diseminate pe teritoriul Noii- } \\
\text { (Delisle 2003, 136). }\end{array}$ \\
\hline
\end{tabular}

Exemple 4:

\begin{tabular}{|l|l|}
\hline \multicolumn{1}{|c|}{ Texte-source } & \multicolumn{1}{c|}{ Texte-cible } \\
\hline $\begin{array}{l}\text { Le 24 juin 1918, a lieu le premier vol } \\
\text { aéropostal officiel au Canada (Delisle } \\
\text { 2003, 137). }\end{array}$ & La 24 iunie 1918, are loc primul zbor \\
aerospațialaeropoștal din Canada. \\
\hline
\end{tabular}

Les syntagmes «maisons de relais » et « vol aéropostal » des exemples 3 et 4 ont été rendus en roumain par " case de schimb» et « zbor aerospațial ». Dans le premier cas, l'apprenti traducteur n'a pas pensé à trouver un équivalent adéquat [ " hanuri de poştă »] qui reflète les pratiques sociales de cette époque-là. Dans le deuxième cas, on a affaire à une faute commise par inadvertance: la ressemblance entre les mots « aéropostal » et " aérospatial » a engendré une reformulation erronée en langue-cible.

Entre la théorie et la pratique traductive, on peut observer un grand décalage. Les étudiants n'arrivent que difficilement à marier savoir et savoir-faire de sorte que le problème que nous rencontrons le plus souvent est la traduction littérale.

Exemple 5:

\begin{tabular}{|l|l|}
\hline \multicolumn{1}{|c|}{ Texte-source } & \multicolumn{1}{|c|}{ Texte-cible } \\
\hline $\begin{array}{l}\text { Les locomotives à vapeur entrent en } \\
\text { service et, dans les années 1880, Van }\end{array}$ & $\begin{array}{l}\text { Locomotivele cu aburi intră în } \\
\text { servieit sunt puse în funcțiune şi, în }\end{array}$ \\
$\begin{array}{l}\text { Horne et ses hommes traversent le } \\
\text { pays en chemin de fer (Delisle 2003, }\end{array}$ & anul 1880, Van Horne şi oamenii săi \\
traversează țara pe cale ferată.
\end{tabular}

\section{Exemple 6:}

\begin{tabular}{|c|c|}
\hline Texte-source & Texte-cible \\
\hline $\begin{array}{l}\text { Ajoutons au passage que la } \\
\text { problématique de la fidélité ne se }\end{array}$ & Să adăugăm pasajului $\quad$ Să \\
\hline
\end{tabular}


pose pas tout à fait dans les mêmes termes pour les textes pragmatiques [...] et les œuvres artistiques (romans, nouvelles, poèmes) (Delisle 2003, 169). problematica fidelității nu se pune pe deplin în aceeaşi termeni pentru textele pragmatice [...] şi operele artistice (romane, nuvele, poeme).

Les syntagmes «entrer en service » et " ajouter au passage » des exemples 5 et 6 ont été traduits littéralement par "a intra în serviciu » et « a adăuga pasajului ». Nous recommandons l'emploi des expressions idiomatiques « a pune în funcțiune » et "a menționa în trecere » que le lecteur roumanophone peut facilement comprendre.

Traduire littéralement est un grand piège pour la plupart des apprentis traducteurs. D'où la nécessité de leur faire comprendre qu'il y a trois paramètres de la fidélité en traduction (Hurtado Albir, 1990): fidélité au vouloir-dire de l'auteur, à la langue-cible et au destinataire. Alors, ils doivent user des ressources de leur langue maternelle et trouver des équivalences qui rendent le texte lisible et cohérent.

La tâche de l'enseignant serait de leur faire prendre conscience de tous les aspects mentionnés ci-dessus et de les aider à choisir une stratégie de traduction adéquate. En ce qui nous concerne, après avoir révisé le texte, lors d'une discussion avec l'étudiant, nous lui expliquons les corrections que nous avons faites et nous lui expliquons aussi pourquoi nous avons considéré nécessaire et utile d'intervenir sur le texte.

\subsection{Rédaction du mémoire de fin d'études}

La deuxième étape de leur travail est la rédaction de la partie introductive et du commentaire de traduction. La méthodologie de recherche et de rédaction du mémoire requiert que les apprentis traducteurs consultent au préalable des ouvrages fondamentaux pour leur domaine d'étude. Nous avons remarqué chez les étudiants la tendance à rédiger des bibliographies très touffues où figurent souvent des livres qui n'ont rien à voir avec le sujet abordé. Puisque nous nous confrontons tous, enseignants et apprentis, à une abondance informationnelle, nous leur apprenons à faire le tri pour ne retenir que les études vraiment utiles et représentatifs pour leur travail. Il est essentiel qu'ils acquièrent une " certaine capacité de discrimination dans la lecture des articles et autres textes de recherche » (Gile 1995, 5). Par exemple, l'apprenti traducteur doit se demander si les faits rapportés «sont suffisamment complets ou représentatifs, s'ils sont pertinents, s'ils sont rapportés correctement, rigoureusement, de manière objective » (Gile 1995, 8). Pouvoir porter un jugement de valeur sur un texte exige que le lecteur détienne certaines connaissances dans le domaine en question. C'est la raison pour laquelle nous considérons nécessaire de guider les étudiants: nous élaguons la bibliographie qu'ils avaient proposée et nous leur faisons des suggestions de lecture. 
Dans l'élaboration de leurs mémoires, les apprentis traducteurs sont amenés à comprendre des documents, à les interpréter et à les reproduire personnellement, mais correctement et avec esprit critique. De même, ils doivent être en mesure d'utiliser une terminologie spécifique pour leur domaine d'étude. Lors de cette étape, nous enseignons aux étudiants à mener une recherche, un travail qui leur soit utile à l'avenir et qu'ils puissent valoriser quel que soit leur futur parcours professionnel.

En ce qui concerne la rédaction des mémoires de licence, un autre problème auquel nous nous trouvons parfois confrontée est le plagiat. Ilie Rad (2008) attire l'attention sur la fréquence de la pratique du plagiat surtout dans le cas des mémoires de licence et l'explique par l'absence prolongée d'une législation en vigueur. C'est en 1996 qu'on a promulgué en Roumanie la loi $\mathrm{N}^{\circ} 8 / 1996$ protégeant les droits d'auteurs et les droits voisins. Malheureusement, cette loi n'empêche pas les emprunts d'idées, de passages, et nous disposons de différents logiciels pour les dépister. Cependant, nous considérons qu'une collaboration étroite de l'enseignant avec l'apprenti traducteur s'avérerait plus utile. Des rencontres fréquentes tout au long du parcours de traduction et de rédaction et des suggestions de la part de l'enseignant aideront les apprentis traducteurs à avoir confiance en leurs propres forces. Il s'agit de les encourager à faire un travail de recherche original même au risque de ne pas obtenir des textes aussi élégants du point de vue de l'expression que ceux qui leur ont servi de point d'appui.

\section{Conclusion}

Rédiger un mémoire de fin d'études permet aux étudiants en traduction et non seulement d'acquérir des compétences spécifiques (expression écrite, expression orale) et de mettre en pratique des aspects théoriques appris pendant les trois années de leur formation universitaire. L'enseignant y joue un rôle important: il aide les apprentis traducteurs à surmonter différents obstacles et il dirige leur recherche en surveillant leur travail et en leur conseillant de consulter des ouvrages de référence pour le sujet abordé.

Dans le cas des mémoires codirigés, nous avons déployé un travail en équipe (enseignant(s)/étudiant) dont les enjeux sont énormes pour le parcours professionnel et personnel de l'apprenti traducteur. Il est nécessaire de stimuler les étudiants à obtenir une certaine confiance et autonomie qui leur serviront dans l'activité professionnelle et dans la vie.

\section{Bibliographie:}

Albir, Amparo Hurtado. La notion de fidélité en traduction. Paris: Didier Erudition, 1990. 
Ballard, Michel. Le commentaire de traduction anglaise. Paris: Armand Colin, [1992] 2005.

Delisle, Jean. La traduction raisonnée. Ottawa: Presses de l'Université d'Ottawa, 2003.

Eco, Umberto. Cum se face o teză de licență [Comment faire un mémoire de licence]. Iaşi: Polirom, 2006.

Gile, Daniel. « La lecture critique en traductologie ». Méta, vol. 40, n 1, 1995: 5-14.

Rad, Ilie. Cum se scrie un text ştiințific. Disciplinele umaniste [Comment écrire un texte scientifique dans le domaine des sciences humaines]. Iaşi: Polirom, 2008.

Rădulescu, Şt. Mihaela. Metodologia cercetării ştiințifice, Elaborarea lucrărilor de licență, masterat, doctorat [Méthodologie de la recherche scientifique, Élaboration des mémoires de licence, de master et des thèses de doctorat]. Bucureşti: Editura didactică şi pedagogică, $2^{\mathrm{e}}$ édition, [2006] 2011.

Țenchea, Maria. « Le rôle du commentaire de traduction dans la formation des traducteurs ». Rodica Superceanu, Daniel Dejica (éds.). Comunicare profesională şi traductologie [Communication professionnelle et traductologie]. Timişoara: Editura Orizonturi Universitare, 2004: 181-186.

This work was cofinaced from the European Social Fund through Sectoral Operational Programme Human Resources Development 2007-2013, project number POSDRU/159/1.5/S/140863, Competitive Researchers in Europe in the Field of Humanities and Socio-Economic Sciences. A Multi-regional Research Network (CCPE) [Cet article a été cofinancé du Fonds Social Européen par le Programme Opérationnel Sectoriel pour le Développement des Ressources Humaines 2007-2013, Code du contrat: POSDRU/159/1.5/S/140863, Chercheurs compétitifs au niveau européen dans le domaine des sciences humaines et socio-économiques. Réseau de recherche multirégional (CCPE)]. 